\title{
One-loop contribution to dark-matter-nucleon scattering in the pseudo-scalar dark matter model
}

\author{
Duarte Azevedo, ${ }^{b}$ Mateusz Duch, ${ }^{a}$ Bohdan Grzadkowski, ${ }^{a}$ Da Huang, ${ }^{a}$ Michal Iglicki ${ }^{a}$ \\ and Rui Santos ${ }^{b}$ \\ ${ }^{a}$ Faculty of Physics, University of Warsaw, \\ Pasteura 5, 02-093 Warsaw, Poland \\ ${ }^{b}$ Centro de Física Teórica e Computacional, Faculdade de Ciências, \\ Universidade de Lisboa, Campo Grande, Edifício C8 1749-016 Lisboa, Portugal \\ E-mail: dazevedo@alunos.fc.ul.pt, mateusz.duch@fuw.edu.pl, \\ bohdan.grzadkowski@fuw.edu.pl, da.huang@fuw.edu.pl, \\ michal.iglicki@fuw.edu.pl, rasantos@fc.ul.pt
}

ABSTRACT: Recent dark matter (DM) direct searches place very stringent constraints on the possible DM candidates proposed in extensions of the Standard Model. There are however models where these constraints are avoided. One of the simplest and most striking examples comes from a straightforward Higgs-portal pseudo-scalar DM model featured with a softly broken U(1) symmetry. In this model the tree-level DM-nucleon scattering cross section vanishes in the limit of zero momentum transfer. It has also been argued that the leading-order DM-nucleon cross section appears at the one-loop level. In this work we have calculated the exact cross section in the zero momentum transfer at the leading order i.e., at the one-loop level of perturbative expansion. We have concluded that, in agreement with expectations, the amplitude for the scattering process is UV finite and approaches zero in the limit of vanishing DM masses. Moreover, we made clear that the finite DM velocity correction at tree level is subdominant with respect to the one-loop contribution. Based on the analytic formulae, our numerical studies show that, for a typical choice of model parameters, the DM nuclear recoiling cross section is well below $\mathcal{O}\left(10^{-50} \mathrm{~cm}^{2}\right)$, which indicates that the DM direct detection signal in this model naturally avoids present strong experimental limits on the cross section.

Keywords: Beyond Standard Model, Higgs Physics

ARXIV EPRINT: 1810.06105 


\section{Contents}

1 Introduction 1

2 The model 2

3 Tree-level contributions to the DM-nucleon scattering at finite DM velocity

4 Explicit calculation of the one-loop DM-nucleon amplitude at zero momentum transfer

4.1 Counterterms and the cancellation of the counterterm-insertion diagrams 6

4.2 Cancellation of SM particle loops 8

$\begin{array}{lll}4.3 & \text { One-loop level DM-nucleon scatterings } & 10\end{array}$

5 Numerical studies $\quad 13$

6 Conclusion 16

$\begin{array}{ll}\text { A Tree-level interacting vertices } & 17\end{array}$

B Analytic expressions of $C$ and $D$ functions 18

\section{Introduction}

The nature of dark matter (DM) [1,2] is still a great mystery of modern physics. Over the last several decades, there have been many experiments to search for DM particles, which focus either on its direct or indirect detection [3, 4]. In particular, the recent XENON1T experiment [5] placed the most stringent upper bound on the DM-nucleon scattering cross section. The limit constitutes a great challenge while constructing DM models.

Some of the simplest realisations of DM are SM extensions with an extra complex scalar field $[6,7]$. In particular there is a model where the complex singlet of the SM gauge group is charged under an extra global $\mathrm{U}(1)$ and for which the real part of the singlet acquires a vacuum expectation value (VEV) [7-12]. Then, the pseudo-Goldstone boson $A$ (imaginary component of the scalar) becomes the massive DM candidate if $\mathrm{U}(1)$ is softly broken and the DM-nucleon $(A N)$ scattering, mediated by the remaining scalars of the theory, the SM-like Higgs and and the extra scalar, can be naturally suppressed if the linear breaking term is removed by a $Z_{2}$ symmetry $[8,10]$. Remarkably, the tree-level DM nuclear recoiling cross section is found to vanish in the limit of zero-momentum-transfer. Recently, it has been argued in ref. [10] that the leading-order contribution to DM nuclear scatterings arises at one-loop order. Based on the analysis of the asymptotic behaviour 
at large and small DM mass, a simple approximate formula for the one-loop DM-nucleon cross sections $\sigma_{\mathrm{AN}}$ was suggested, which implies that the natural value of $\sigma_{\mathrm{AN}}$ should be much smaller than the current experimental upper limits. This result shows that the DM direct detection experiments do not constrain the model, which is explicitly demonstrated in ref. [12] by scanning the whole parameter space.

However, there are still several potential problems plaguing the above conclusion. Firstly, both the final results given in ref. [10] and the scans shown in ref. [12] depend crucially on the aforementioned approximation. One natural question is how accurate this simple approximation is. Secondly, beyond the zero-momentum-transfer limit, the DMnucleon cross section is nonzero with the correction coming from the finite momentum transfer. What is the typical order of this finite-momentum-transfer correction? Which contribution is dominant, the tree-level cross section or one-loop one? Furthermore, it is expected that increasingly smaller scattering cross sections will be probed by the next generation planned experiments XENONnT [13], LZ [14] and DARWIN [15] (see also [16]), which imply a demand for increasing precision in the theoretical calculations. Therefore, in order to answer the above questions, and to be prepared for the future experimental results on direct detection, we need to explicitly calculate both contributions analytically and numerically, which is the main motivation to the present work. A similar strategy, although in a context of a different model, has been considered very recently in ref. [17].

The paper is organised as follows. In section 2, we briefly introduce the model and define our notation and conventions. Section 3 is devoted to the calculations of finite treelevel contributions coming from the finite DM velocity or finite momentum transfer. In section 4, we show the analytic expression of the one-loop DM-nucleon cross section in the limit of zero momentum transfer. Then we show our numerical studies in section 5 . Finally, the short summary is given in section 6 .

\section{The model}

We begin our discussion by specifying the Higgs-portal complex scalar DM model [10, 12]. The SM is extended by an extra complex scalar singlet $S$ which possesses an intrinsic global $\mathrm{U}(1)$ symmetry $S \rightarrow e^{i \alpha} S$. Then we softly break this dark U(1) symmetry to the residual $Z_{2}$ symmetry $S \rightarrow-S$ via a mass term $\mu^{2} S^{2}+$ H.c.. Thus, the scalar potential is given by

$$
\begin{aligned}
\mathcal{V}= & -\mu_{H}^{2}|H|^{2}-\mu_{S}^{2}|S|^{2}+\lambda_{H}|H|^{4}+\lambda_{S}|S|^{4}+\kappa|H|^{2}|S|^{2} \\
& +\left(\mu^{2} S^{2}+\text { H.c. }\right)
\end{aligned}
$$

where $H$ denotes the SM Higgs doublet. Note that we can make $\mu^{2}$ real by rotating the phase of $S$. As a result, an additional dark $C P$ symmetry $S \rightarrow S^{*}$ of the potential (2.1) emerges. Thus, the total symmetry of this model is $Z_{2} \times C P$. Note also that the $\mathrm{SM}$ and the scalar $S$ are coupled to each other only via the quartic scalar coupling $\kappa|H|^{2}|S|^{2}$, which is the prominent feature of Higgs portal models. We will consider the case in which the scalars $H$ and $S$ have non-zero vacuum expectation values (VEVs), $\langle H\rangle=\left(0, v_{H} / \sqrt{2}\right)^{T}$ and $\langle S\rangle=v_{S} / \sqrt{2}$. By minimizing the scalar potential in eq. (2.1), we obtain the following 
two conditions

$$
\begin{aligned}
-\mu_{H}^{2}+\lambda_{H} v_{H}^{2}+\frac{1}{2} \kappa v_{S}^{2} & =0, \\
-\left(\mu_{S}^{2}-2 \mu^{2}\right)+\lambda_{S} v_{S}^{2}+\frac{1}{2} \kappa v_{H}^{2} & =0,
\end{aligned}
$$

which have the solution

$$
v_{H}^{2}=\frac{(\kappa / 2) \mu_{H}^{2}-\lambda_{H}\left(\mu_{S}^{2}-2 \mu^{2}\right)}{\left(\kappa^{2} / 4\right)-\lambda_{H} \lambda_{S}}, \quad v_{S}^{2}=\frac{(\kappa / 2)\left(\mu_{S}^{2}-2 \mu^{2}\right)-\lambda_{S} v_{H}^{2}}{\left(\kappa^{2} / 4\right)-\lambda_{H} \lambda_{S}} .
$$

These are the conditions for the EW gauge and $Z_{2}$ symmetries to be broken spontaneously. We will be working in the unitary gauge where the scalars fields are written as

$$
H=\left(\begin{array}{c}
0 \\
\left(v_{H}+h\right) / \sqrt{2}
\end{array}\right), \quad S=\frac{v_{S}+s+i A}{\sqrt{2}} .
$$

Since the U(1) symmetry is softly broken, the would-be Goldstone boson $A$ becomes massive, with a mass given by $m_{A}^{2}=-4 \mu^{2}$. On the other hand, this particle is odd under the preserved dark $C P$ symmetry, which guarantees its stability so that it can be the DM candidate. The other two $C P$-even scalar components $h$ and $s$, with their mass-squared matrix given by

$$
\mathcal{M}^{2}=\left(\begin{array}{cc}
2 \lambda_{H} v_{H}^{2} & \kappa v_{H} v_{S} \\
\kappa v_{H} v_{S} & 2 \lambda_{S} v_{S}^{2}
\end{array}\right)
$$

mix via the following orthogonal transformation to form the mass eigenstates $h_{1,2}$ as

$$
\left(\begin{array}{c}
h \\
s
\end{array}\right)=\left(\begin{array}{cc}
c_{\alpha} & -s_{\alpha} \\
s_{\alpha} & c_{\alpha}
\end{array}\right)\left(\begin{array}{l}
h_{1} \\
h_{2}
\end{array}\right),
$$

where $s_{\alpha} \equiv \sin \alpha, c_{\alpha} \equiv \cos \alpha$ and $\alpha$ is the mixing angle. Denoting the masses of $h_{1,2}$ as $m_{1,2}$, we have the following relations

$$
\lambda_{H}=\frac{c_{\alpha}^{2} m_{1}^{2}+s_{\alpha}^{2} m_{2}^{2}}{2 v_{H}^{2}}, \quad \lambda_{S}=\frac{s_{\alpha}^{2} m_{1}^{2}+c_{\alpha}^{2} m_{2}^{2}}{2 v_{S}^{2}}, \quad \kappa=\frac{s_{\alpha} c_{\alpha}\left(m_{1}^{2}-m_{2}^{2}\right)}{v_{H} v_{S}} .
$$

In the following, we will assume that $h_{1}$ is the SM-like Higgs already discovered at the LHC, with a mass of $125 \mathrm{GeV}$, while $h_{2}$ is the other CP-even scalar which, we already know, is mainly singlet-like since the bound on the mixing angle is at present of the order $|\sin \alpha| \leq$ 0.35 and it come from the combined signal strength measurements of the production and decay of the SM-like Higgs, $h_{1}$ [18]. We choose as input parameters for our study $m_{1,2}, s_{\alpha}$, $v_{H, S}$ and $m_{A}$. The triple- and quartic-scalar terms in the scalar-potential eq. (2.1) generate interaction vertices, which are listed in appendix A. 


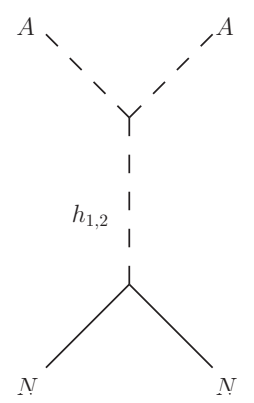

Figure 1. Tree-level diagrams for DM-nucleon scattering.

\section{Tree-level contributions to the DM-nucleon scattering at finite DM velocity}

At the tree level, there are only two diagrams with $h_{1,2}$ exchange displayed in figure 1 , the corresponding amplitude for the DM-nucleon scattering was shown in refs. $[10,12]$ to vanish in the limit of zero momentum transfer. However, a non-vanishing DM-nucleon cross section can be obtained when we consider the finite DM velocity in the rest frame of the DM detector, although its size will be shown to be much smaller than the one-loop quantum contribution presented in the following sections.

The total amplitude for the DM-nucleon interaction at the tree level is given by

$$
\begin{aligned}
-i \mathcal{M}_{\text {tree }} & =-\frac{i 2 f_{N} m_{N}}{v_{H}}\left(\frac{V_{A A 1} c_{\alpha}}{q^{2}-m_{1}^{2}}-\frac{V_{A A 2} s_{\alpha}}{q^{2}-m_{2}^{2}}\right) \bar{u}_{N}\left(p_{4}\right) u_{N}\left(p_{2}\right) \\
& =-i \frac{s_{\alpha} c_{\alpha} f_{N} m_{N}}{v_{H} v_{S}}\left(\frac{m_{1}^{2}}{q^{2}-m_{1}^{2}}-\frac{m_{2}^{2}}{q^{2}-m_{2}^{2}}\right) \bar{u}_{N}\left(p_{4}\right) u_{N}\left(p_{2}\right) \\
& \approx-i \frac{s_{\alpha} c_{\alpha} f_{N} m_{N}}{v_{H} v_{S}}\left(\frac{m_{1}^{2}-m_{2}^{2}}{m_{1}^{2} m_{2}^{2}}\right) q^{2} \bar{u}_{N}\left(p_{4}\right) u_{N}\left(p_{2}\right),
\end{aligned}
$$

where $q^{2}$ is the DM momentum transfer when it scatters against nucleons. Here $m_{N}$ and $f_{N} \approx 0.3$ represent the nucleon mass and its SM Higgs coupling [19-21], respectively. In the third line, we have only kept the leading-order dependence on the momentum transfer. Therefore, the tree-level cross section $\sigma_{\mathrm{AN}}^{\text {tree }}$ is given by

$$
\sigma_{\mathrm{AN}}^{\text {tree }} \approx \frac{4 s_{\alpha}^{2} c_{\alpha}^{2} f_{N}^{2}}{3 \pi} \frac{m_{N}^{2} \mu_{\mathrm{AN}}^{6}}{m_{A}^{2} v_{H}^{2} v_{S}^{2}} \frac{\left(m_{1}^{2}-m_{2}^{2}\right)^{2}}{m_{1}^{4} m_{2}^{4}} v_{A}^{4}
$$

where $\mu_{\mathrm{AN}} \equiv m_{A} m_{N} /\left(m_{A}+m_{N}\right)$ is the reduced mass in the DM-nucleon system, and $v_{A}$ is the DM velocity in the lab frame. Note that the typical relative velocity of a DM particle in the vicinity of the Earth is expected to be $v_{A} \sim 200 \mathrm{~km} / \mathrm{s}$, which would suppress the $\mathrm{DM}$ nuclear recoil cross section by a factor of order of $v_{A}^{4} \sim 10^{-13}$. If we adopt typical values of parameters, say $v_{S}=1 \mathrm{TeV}, m_{2}=300 \mathrm{GeV}, s_{\alpha}=0.1$ and $m_{A}=100 \mathrm{GeV}$, then the tree-level cross section is estimated as $\sigma_{\mathrm{AN}}^{\text {tree }}=7.6 \sim 10^{-68} \mathrm{~cm}^{2}$, which is too small to be observed experimentally at present but also in the future planned experiments. 


\section{Explicit calculation of the one-loop DM-nucleon amplitude at zero momentum transfer}

In this section, we are going to calculate the one-loop contributions to the DM-nucleon scattering cross section. We would like to first argue that some diagrams that would in principle contribute to the scattering amplitude, can be omitted because they are subdominant. At the fundamental level, the DM-nucleon scattering can be understood as the scattering of the DM particle $A$ with light quarks, $q=u, d, s$, and gluons. Since the light quark Yukawa couplings are extremely small, the diagrams with multiple insertions of light quark Yukawa couplings, exemplified in diagrams (a) and (b) in figure 2, are expected to be negligibly small. Hence, it is sufficient for the required precision to keep only diagrams with only one light quark Yukawa coupling insertion. Also, it is easy to show that the oneloop corrections to the external quark lines and the vertices $h_{i} \bar{q} q$ are always proportional to their tree-level counterparts, which means that they are canceled identically in the limit of zero momentum transfer. Therefore, the remaining diagrams for DM-quark scattering can be viewed as the one-loop vertex corrections to $A A h_{1}$ and $A A h_{2}$. On the other hand, the DM nuclear recoils can also be induced by the DM-gluon scattering, for which the next-to-leading-order contribution emerges at the two-loop level. In contrast to the quark case, the diagrams like the one in figure 2(c) with two internal Higgs lines attached to the top loop, should be of the same order as the two-loop ones with only one Higgs coupling to the top loop, since the top quark Yukawa coupling is of $\mathcal{O}(1)$. Nevertheless, in the present paper, we restrict ourselves to the calculation of diagrams with only one Higgs coupling to the top-quark loop, assuming that other diagrams with double Higgs coupling should be much smaller. Actually, based on the computations in ref. [22], we have good reasons to expect that this is indeed the case. Concluding, we are going to focus on the diagrams with only a single Higgs Yukawa coupling either to an external light quark line (for DM-light quark scattering) or to a loop top quark line (for DM-gluon scattering). Therefore we can reduce our calculation to the one-loop corrections $V_{A A 1, A A 2}^{(1)}$ to the vertices $A A h_{1}$ and $A A h_{2}$, respectively, combining the Higgs-quark and Higgs-gluon couplings to a nucleon into a single Higgs-nucleon-nucleon form factor $f_{N} m_{N} / v_{H}$, as we did for the tree-level diagrams in section 3 .

Furthermore, we will work in the limit of zero momentum transfer $q^{2} \rightarrow 0$ in order to simplify our calculation, which is justified by the fact that the terms proportional to $q^{2}$ are suppressed further by powers of the relative DM velocities as was previously illustrated in the case of the tree-level computations. As a result, the one-loop contributions to the DM nuclear recoil reactions in the present model can be represented as

$$
\sigma_{\mathrm{AN}}^{(1)}=\frac{f_{N}^{2}}{\pi v_{H}^{2}} \frac{m_{N}^{2} \mu_{\mathrm{AN}}^{2}}{m_{A}^{2}} \mathcal{F}^{2}
$$

where the one-loop function $\mathcal{F}$ is defined as

$$
\mathcal{F}=\frac{V_{A A 1}^{(1)} c_{\alpha}}{m_{1}^{2}}-\frac{V_{A A 2}^{(1)} s_{\alpha}}{m_{2}^{2}}
$$




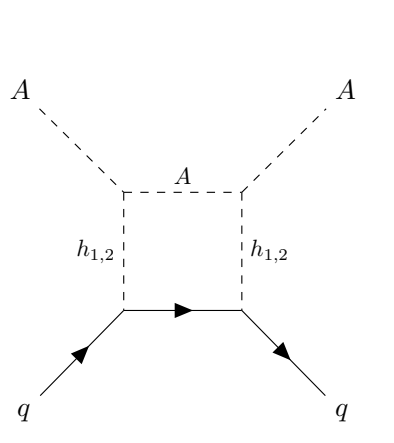

(a)

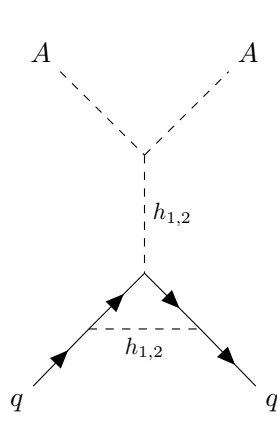

(b)

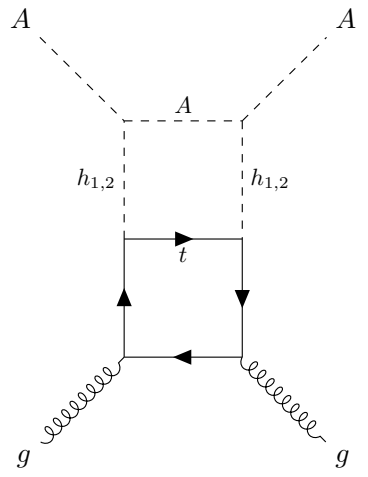

(c)

Figure 2. Examples of diagrams contributing to DM-nucleon scattering, which are discarded in our computation. Diagrams (a) and (b) represent the one-loop box and light-quark- $h_{1,2}$ vertex corrected diagrams which are ignored due to the multiple Yukawa coupling suppression, while the diagram (c) is an example of DM-gluon scattering with two Higgs lines inserted into the top-quark loop, which is assumed to be subdominant.

with $V_{A A 1, A A 2}^{(1)}$ as the aforementioned one-loop corrections to the vertices $h_{1} A^{2}$ and $h_{2} A^{2}$. Therefore, our main task in the present section is to calculate the function $\mathcal{F}$ and associated $V_{A A 1, A A 2}^{(1)}$.

The above one-loop function, $\mathcal{F}$, should satisfy two consistency conditions. Firstly, since the tree-level $A N$ recoiling amplitude vanishes in the limit of zero momentum transfer, the one-loop amplitude and $\mathcal{F}$ should be finite in the same limit. In other words, we do not need to renormalise the model, that is, although we will define a set of counterterms, it will be shown that no renormalisation prescription is needed because the set of diagrams with counterterms only is zero. Consequently, the sum of all diagrams has to be finite. Secondly, in the limit of $m_{A}^{2}=-4 \mu^{2} \rightarrow 0$, the dark matter particle $A$ would return to its true Goldstone boson nature due to the spontaneous breaking of the global U(1) symmetry. In this limit, it is argued in ref. [10] that the corresponding $A N$ scattering amplitude should be only proportional to $q^{2}$ and thus it should vanish when $q^{2} \rightarrow 0$. This indicates that $\mathcal{F}$ should approach zero in the limit $m_{A}^{2} \rightarrow 0$. These observations are two important criteria, which are useful to check the correctness of our final result.

\subsection{Counterterms and the cancellation of the counterterm-insertion diagrams}

Before delving into the calculation of the one-loop diagrams, we firstly show the cancellation of the counterterm-insertion diagrams in figure 3. In order to do that, we need to specify some relations among the counterterms in the present DM model.

The model has 6 independent parameters, as can be seen from eq. (2.1) that defines the potential, and therefore we need 6 counterterms to cancel the UV divergences at the one-loop order. Note that since we will show that we do not need a renormalisation prescription, we refrain from discussing the complete set of renormalisation constants in the Lagrangian. The contribution from the remaining SM terms will be discussed later. We have two methods to construct such counterterms. One is to work with the original 


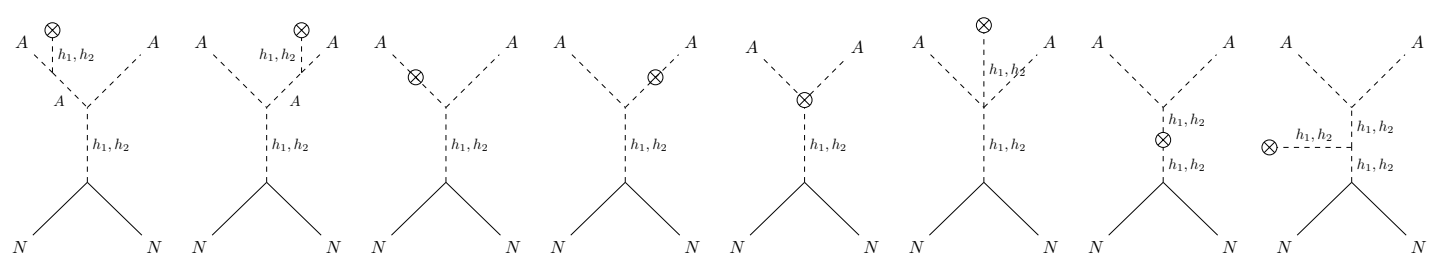

Figure 3. Counterterm-insertion diagrams.

Lagrangian parameters in eq. (2.1) with the following counterterms

$$
\mathcal{V}_{c}=-\delta \mu_{H}^{2}|H|^{2}-\delta \mu_{S}^{2}|S|^{2}+\delta \lambda_{H}|H|^{4}+\delta \lambda_{S}|S|^{4}+\delta \kappa|H|^{2}|S|^{2}+\left(\delta \mu^{2} S^{2}+\text { H.c. }\right),
$$

with the same form as the original potential with the subscript $c$ labelling counterterms. Here we have assumed that the parameters in eq. (2.1) correspond to the renormalised quantities. Furthermore, as we shall see below, we do not need the field wave function renormalisation counterterms since their contributions either vanish in the limit of zero momentum transfer or are cancelled in the computations. ${ }^{1}$ The other way is to define them in terms of the physical mass eigenstates $h_{1,2}$ and $A$, by writing the following potential terms up to quadratic ones

$$
\mathcal{V}_{c}^{(2)}=\delta t_{1} h_{1}+\delta t_{2} h_{2}+\frac{1}{2} \delta m_{1}^{2} h_{1}^{2}+\frac{1}{2} \delta m_{2}^{2} h_{2}^{2}+\delta m_{12}^{2} h_{1} h_{2}+\frac{1}{2} \delta m_{A}^{2} A^{2} .
$$

These two sets of counterterms can be related to each other by expanding eq. (4.3) in terms of the mass eigenstates, eq. (2.6), up to quadratic order in fields. The original set can be written in terms of the new set of parameters as

$$
\begin{aligned}
\delta \mu_{H}^{2}= & \frac{1}{2}\left(c_{\alpha}^{2} \delta m_{1}^{2}+s_{\alpha}^{2} \delta m_{2}^{2}-2 s_{\alpha} c_{\alpha} \delta m_{12}^{2}\right) \\
& +\frac{v_{S}}{2 v_{H}}\left[s_{\alpha} c_{\alpha}\left(\delta m_{1}^{2}-\delta m_{2}^{2}\right)+\left(c_{\alpha}^{2}-s_{\alpha}^{2}\right) \delta m_{12}^{2}\right]-\frac{3}{2 v_{H}}\left(\delta t_{1} c_{\alpha}-\delta t_{2} s_{\alpha}\right), \\
\delta \mu_{S}^{2}= & \frac{1}{2}\left(s_{\alpha}^{2} \delta m_{1}^{2}+c_{\alpha}^{2} \delta m_{2}^{2}+2 s_{\alpha} c_{\alpha} \delta m_{12}^{2}-\delta m_{A}^{2}\right) \\
& +\frac{v_{H}}{2 v_{S}}\left[s_{\alpha} c_{\alpha}\left(\delta m_{1}^{2}-\delta m_{2}^{2}\right)+\left(c_{\alpha}^{2}-s_{\alpha}^{2}\right) \delta m_{12}^{2}\right]-\frac{1}{v_{S}}\left(\delta t_{1} s_{\alpha}+\delta t_{2} c_{\alpha}\right), \\
\delta \mu^{2}= & \frac{1}{4 v_{S}}\left(\delta t_{1} s_{\alpha}+\delta t_{2} c_{\alpha}\right)-\frac{1}{4} \delta m_{A}^{2} \\
\delta \kappa= & \frac{1}{v_{H} v_{S}}\left[s_{\alpha} c_{\alpha}\left(\delta m_{1}^{2}-\delta m_{2}^{2}\right)+\left(c_{\alpha}^{2}-s_{\alpha}^{2}\right) \delta m_{12}^{2}\right] \\
\delta \lambda_{H}= & \frac{1}{2 v_{H}^{2}}\left(c_{\alpha}^{2} \delta m_{1}^{2}+s_{\alpha}^{2} \delta m_{2}^{2}-2 s_{\alpha} c_{\alpha} \delta m_{12}^{2}\right)-\frac{1}{2 v_{H}^{3}}\left(\delta t_{1} c_{\alpha}-\delta t_{2} s_{\alpha}\right), \\
\delta \lambda_{S}= & \frac{1}{2 v_{S}^{2}}\left(s_{\alpha}^{2} \delta m_{1}^{2}+c_{\alpha}^{2} \delta m_{2}^{2}+2 s_{\alpha} c_{\alpha} \delta m_{12}^{2}\right)-\frac{1}{2 v_{S}^{3}}\left(\delta t_{1} s_{\alpha}+\delta t_{2} c_{\alpha}\right) .
\end{aligned}
$$

Now we proceed to compute the total contribution from the counterterm-insertion diagrams shown in figure 3 . Note that the diagrams with external $A$-line corrections imply

\footnotetext{
${ }^{1}$ In fact, we could have opted to work with unrenormalised fields [23] which gives rise to Green functions that are in general divergent but leads to finite S-matrix elements.
} 
the following contributions to $\mathcal{F}$

$$
\mathcal{F}_{c e}=-2\left(\delta_{A} p^{2}-\delta m_{A}^{2}+\frac{2 V_{A A 1} \delta t_{1}}{m_{1}^{2}}+\frac{2 V_{A A 2} \delta t_{2}}{m_{2}^{2}}\right) \frac{1}{p^{2}-m_{A}^{2}} \mathcal{F}_{0}=0,
$$

where the subscript $e$ represents the external DM lines. Here $\delta_{A}$ is the DM $A$ wave function counterterm and $p^{2}$ is its momentum, and

$$
\mathcal{F}_{0}=\frac{V_{A A 1} c_{\alpha}}{m_{1}^{2}}-\frac{V_{A A 2} s_{\alpha}}{m_{2}^{2}}
$$

is the tree-level counterpart of $\mathcal{F}$ which appears in the first equality of eq. (3.1) in the limit of zero momentum transfer. Note that $\mathcal{F}_{0}=0$ if we apply the tree-level relations in eq. (2.7), which leads to the vanishing $\mathcal{F}_{c e}$. For the remaining diagrams in figure 3 , we can calculate their contributions to the effective vertices $A A h_{1}$ and $A A h_{2}$ directly as

$$
\begin{aligned}
-i V_{A A 1 c(i+v)}^{(1)}= & i V_{A A 1} \frac{\delta m_{1}^{2}}{m_{1}^{2}}+i V_{A A 2} \frac{\delta m_{12}^{2}}{m_{2}^{2}} \\
& -\frac{6 i V_{A A 1} V_{111} \delta t_{1}}{m_{1}^{4}}-\frac{2 i V_{A A 1} V_{112} \delta t_{2}}{m_{1}^{2} m_{2}^{2}}-\frac{2 i V_{A A 2} V_{112} \delta t_{1}}{m_{1}^{2} m_{2}^{2}}-\frac{2 i V_{A A 2} V_{122} \delta t_{2}}{m_{2}^{4}} \\
& +\frac{2 i V_{A A 11} \delta t_{1}}{m_{1}^{2}}+\frac{i V_{A A 12} \delta t_{2}}{m_{2}^{2}}-i\left(s_{\alpha} v_{S} \delta \lambda_{S}+\frac{1}{2} c_{\alpha} v_{H} \delta \kappa\right), \\
-i V_{A A 2 c(i+v)}^{(1)}= & i V_{A A 1} \frac{\delta m_{12}^{2}}{m_{1}^{2}}+i V_{A A 2} \frac{\delta m_{2}^{2}}{m_{2}^{2}} \\
& -\frac{2 i V_{A A 1} V_{112} \delta t_{1}}{m_{1}^{4}}-\frac{2 i V_{A A 1} V_{122} \delta t_{2}}{m_{1}^{2} m_{2}^{2}}-\frac{2 i V_{A A 2} V_{122} \delta t_{1}}{m_{1}^{2} m_{2}^{2}}-\frac{6 i V_{A A 2} V_{222} \delta t_{2}}{m_{2}^{4}} \\
& +\frac{i V_{A A 12} \delta t_{1}}{m_{1}^{2}}+\frac{2 i V_{A A 22} \delta t_{2}}{m_{2}^{2}}-i\left(c_{\alpha} v_{S} \delta \lambda_{S}-\frac{1}{2} s_{\alpha} v_{H} \delta \kappa\right),
\end{aligned}
$$

where the subscripts $i$ and $v$ denote the corrections to internal $h_{1,2}$ propagators and $h_{1,2} A^{2}$ vertices, respectively. We also set the four-momenta of the internal $h_{1,2}$ lines to be zero since the momentum transfer vanishes by assumption. In each equation in (4.8), the first two lines correspond to the internal $h_{1,2}$-propagator corrections, while the third line to the vertices $A A h_{1}$ and $A A h_{2}$ corrections. With these two expressions, we can show their contributions to $\mathcal{F}$ vanishes

$$
\mathcal{F}_{c(i+v)}=\frac{V_{A A 1 c b}^{(1)} c_{\alpha}}{m_{1}^{2}}-\frac{V_{A A 2 c b}^{(1)} s_{\alpha}}{m_{2}^{2}}=0,
$$

where we have used the relations in eq. (4.5) to represent the dimensionless coupling counterterms $\delta \kappa, \delta \lambda_{S}$, and $\delta \lambda_{H}$ in terms of the physical set. We have also employed the definitions of the tree-level vertices eq. (A.1) and the relations in eq. (2.7).

\subsection{Cancellation of SM particle loops}

In this subsection, we will show that the one-loop contributions from the SM particle loops other than the Higgs cancel. For illustration purposes we will adopt the top-quark loops in figure 4 to show the main features of this cancellation. Note that the remaining SM 

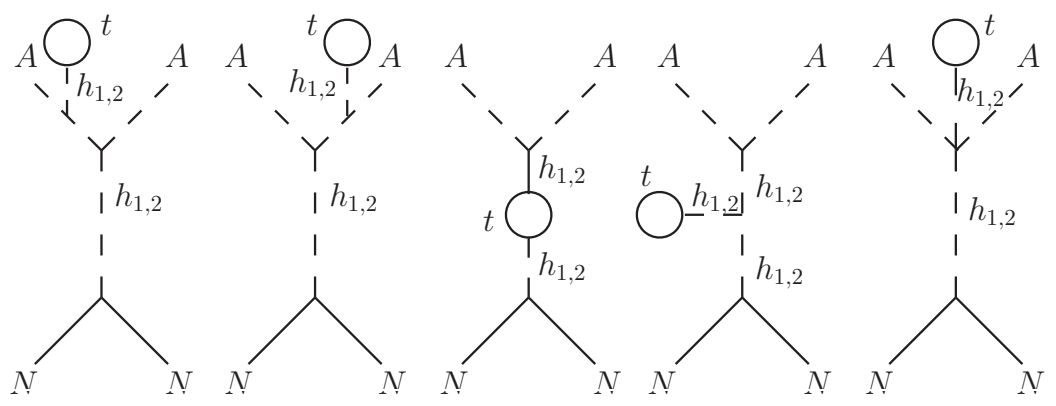

Figure 4. Top-quark-loop diagrams for DM-nucleon scatterings.

particles, quarks, leptons, and electroweak gauge bosons, couple to the Higgs bosons $h_{1,2}$ only through the rotation of the doublet neutral components $h$ with the couplings given by

$$
g_{\eta 1}=g_{\eta} c_{\alpha}, \quad g_{\eta 2}=-g_{\eta} s_{\alpha},
$$

where $g_{\eta}$ represents the SM particle species $\eta$ coupling to the original SM Higgs $h$. For the top quark, its couplings to $h_{1,2}$ are $y_{t 1}=y_{t} c_{\alpha}$ and $y_{t 2}=-y_{t} s_{\alpha}$, respectively. Moreover, it can be seen from figure 4 that the SM loops can appear in corrections via the Higgs bosons tadpoles, either connected to the dark matter particle A or to another Higgs line, or via two-point functions, which are corrections to the Higgs propagators or finally as corrections to vertices. For these three contributions, the top-quark-loop $A A h_{1}$ and $A A h_{2}$ corrections are given by

$$
\begin{aligned}
-i V_{A A 1 e}^{(1)}= & -\frac{2 V_{A A 1}}{p^{2}-m_{A}^{2}}\left(\frac{V_{A A 1} c_{\alpha}}{m_{1}^{2}}+\frac{V_{A A 2} s_{\alpha}}{m_{s}^{2}}\right) L_{1}, \\
-i V_{A A 2 e}^{(1)}= & -\frac{2 V_{A A 2}}{p^{2}-m_{A}^{2}}\left(\frac{V_{A A 1} c_{\alpha}}{m_{1}^{2}}+\frac{V_{A A 2} s_{\alpha}}{m_{s}^{2}}\right) L_{1}, \\
-i V_{A A 1 i}^{(1)}= & -\left(\frac{V_{A A 1} c_{\alpha}^{2}}{m_{1}^{2}}-\frac{V_{A A 2} c_{\alpha} s_{\alpha}}{m_{2}^{2}}\right) L_{2} \\
& +\left(\frac{6 V_{A A 1} V_{111} c_{\alpha}}{m_{1}^{4}}-\frac{2 V_{A A 1} V_{112} s_{\alpha}}{m_{1}^{2} m_{2}^{2}}+\frac{2 V_{A A 2} V_{112} c_{\alpha}}{m_{1}^{2} m_{2}^{2}}-\frac{2 V_{A A 2} V_{122} s_{\alpha}}{m_{2}^{4}}\right) L_{1}, \\
-i V_{A A 2 i}^{(1)}= & -\left(-\frac{V_{A A 1} s_{\alpha} c_{\alpha}}{m_{1}^{2}}+\frac{V_{A A 2} s_{\alpha}^{2}}{m_{2}^{2}}\right) L_{2} \\
& +\left(\frac{2 V_{A A 1} V_{112} c_{\alpha}}{m_{1}^{4}}-\frac{2 V_{A A 1} V_{122} s_{\alpha}}{m_{1}^{2} m_{2}^{2}}+\frac{2 V_{A A 2} V_{122} c_{\alpha}}{m_{1}^{2} m_{2}^{2}}-\frac{6 V_{A A 2} V_{222} s_{\alpha}}{m_{2}^{4}}\right) L_{1}, \\
-i V_{A A 1 v}^{(1)}= & -\left(\frac{2 V_{A A 11} c_{\alpha}}{m_{1}^{2}}-\frac{V_{A A 12} s_{\alpha}}{m_{2}^{2}}\right) L_{1}, \\
-i V_{A A 2 v}^{(1)}= & -\left(\frac{V_{A A 12} c_{\alpha}}{m_{1}^{2}}-\frac{2 V_{A A 22} s_{\alpha}}{m_{2}^{2}}\right) L_{1},
\end{aligned}
$$

where, for top quarks, the tadpole and bubble one-loop integrals can be represented as follows

$$
\begin{aligned}
& L_{1}=(-1)\left(-i y_{t}\right) \int \frac{d^{4} l}{(2 \pi)^{4}} \operatorname{Tr}\left[\frac{i}{l-m_{t}}\right], \\
& L_{2}=(-1)\left(-i y_{t}\right)^{2} \int \frac{d^{4} l}{(2 \pi)^{4}} \operatorname{Tr}\left[\frac{i^{2}}{\left(\not-m_{t}\right)^{2}}\right],
\end{aligned}
$$


where $\operatorname{Tr}$ denotes the trace over the spinor space. With $V_{A A 1(A A 2) e}^{(1)}$, it is easy to write down the following contribution to $\mathcal{F}$ from the external $A$ correction

$$
\mathcal{F}_{e}=(-i) \frac{2 L_{1}}{p^{2}-m_{A}^{2}}\left(\frac{V_{A A 1} c_{\alpha}}{m_{1}^{2}}+\frac{V_{A A 2} s_{\alpha}}{m_{2}^{2}}\right) \mathcal{F}_{0}=0
$$

in which the second equality follows from the identity $\mathcal{F}_{0}=0$. For the remaining diagrams, we can apply the definitions of the tree-level couplings in appendix A and the tree-level relations in eq. (2.7) to directly prove

$$
\mathcal{F}_{i+v}=\frac{\left(V_{A A 1 i}^{(1)}+V_{A A 1 v}^{(1)}\right) c_{\alpha}}{m_{1}^{2}}-\frac{\left(V_{A A 2 i}^{(1)}+V_{A A 2 v}^{(1)}\right) s_{\alpha}}{m_{2}^{2}}=0 .
$$

In the above derivation, what is crucial for the cancellation is the dependence of topquark Yukawa couplings on the mixing angle $\alpha$. Since for a given Higgs boson $h_{i}$ the mixing matrix enters the same way for all SM fermions and electroweak gauge bosons, therefore the cancellation is present for all SM particles (except $h_{1,2}$ ) in the loops as well.

\subsection{One-loop level DM-nucleon scatterings}

Having proved the cancellation of all diagrams involving the counterterms and the SM particle loops, we now focus on loop diagrams generated by the Higgs bosons $h_{1,2}$ and the scalar DM particle $A$. As shown below, we can divide these one-loop diagrams into three classes: the corrections to the external DM lines $A$, to the vertices $V_{A A 1, A A 2}$, and to the internal Higgs propagators. Note that all expression will be written as a function of the triple- and quartic-scalar terms in the scalar-potential eq. (2.1) listed in appendix A. It is useful to first define the following one-particle irreducible (1PI) one-loop diagrams.

- The $h_{1,2}$ and $A$ tadpole corrections:

$$
\begin{aligned}
& -i \Delta t_{1}=\int \frac{d^{4} l}{(2 \pi)^{4}}\left(\frac{3 V_{111}}{l^{2}-m_{1}^{2}}+\frac{V_{122}}{l^{2}-m_{2}^{2}}+\frac{V_{A A 1}}{l^{2}-m_{A}^{2}}\right), \\
& -i \Delta t_{2}=\int \frac{d^{4} l}{(2 \pi)^{4}}\left(\frac{V_{112}}{l^{2}-m_{1}^{2}}+\frac{3 V_{222}}{l^{2}-m_{2}^{2}}+\frac{V_{A A 2}}{l^{2}-m_{A}^{2}}\right),
\end{aligned}
$$

- The $h_{1,2}$ and $A$ mass-squared corrections:

$$
\begin{aligned}
-i \Delta m_{1}^{2}= & \int \frac{d^{4} l}{(2 \pi)^{4}}\left[\frac{18 V_{111}^{2}}{\left(l^{2}-m_{1}^{2}\right)^{2}}+\frac{4 V_{112}^{2}}{\left(l^{2}-m_{1}^{2}\right)\left(l^{2}-m_{2}^{2}\right)}+\frac{2 V_{122}^{2}}{\left(l^{2}-m_{2}^{2}\right)^{2}}+\frac{2 V_{A A 1}^{2}}{\left(l^{2}-m_{A}^{2}\right)^{2}}\right] \\
& +\left[\frac{12 V_{1111}}{l^{2}-m_{1}^{2}}+\frac{2 V_{1122}}{l^{2}-m_{2}^{2}}+\frac{2 V_{A A 11}}{l^{2}-m_{A}^{2}}\right] \\
-i \Delta m_{2}^{2}= & \int \frac{d^{4} l}{(2 \pi)^{4}}\left[\frac{2 V_{112}^{2}}{\left(l^{2}-m_{1}^{2}\right)^{2}}+\frac{4 V_{122}^{2}}{\left(l^{2}-m_{1}^{2}\right)\left(l^{2}-m_{2}^{2}\right)}+\frac{18 V_{222}^{2}}{\left(l^{2}-m_{2}^{2}\right)^{2}}+\frac{2 V_{A A 2}^{2}}{\left(l^{2}-m_{A}^{2}\right)^{2}}\right] \\
& +\left[\frac{2 V_{1122}}{l^{2}-m_{1}^{2}}+\frac{12 V_{2222}}{l^{2}-m_{2}^{2}}+\frac{2 V_{A A 22}}{l^{2}-m_{A}^{2}}\right], \\
-i \Delta m_{12}^{2}= & \int \frac{d^{4} l}{(2 \pi)^{4}}\left[\frac{6 V_{111} V_{112}}{\left(l^{2}-m_{1}^{2}\right)^{2}}+\frac{4 V_{112} V_{122}}{\left(l^{2}-m_{1}^{2}\right)\left(l^{2}-m_{2}^{2}\right)}+\frac{6 V_{122} V_{222}}{\left(l^{2}-m_{2}^{2}\right)^{2}}+\frac{2 V_{A A 1} V_{A A 2}}{\left(l^{2}-m_{A}^{2}\right)^{2}}\right]
\end{aligned}
$$




$$
\begin{aligned}
& +\left[\frac{3 V_{1112}}{l^{2}-m_{1}^{2}}+\frac{3 V_{1222}}{l^{2}-m_{2}^{2}}+\frac{V_{A A 12}}{l^{2}-m_{A}^{2}}\right] \\
-i \Delta m_{A}^{2}= & \int \frac{d^{4} l}{(2 \pi)^{4}}\left[\frac{4 V_{A A 1}^{2}}{\left[(l+p)^{2}-m_{A}^{2}\right]\left(l^{2}-m_{1}^{2}\right)}+\frac{4 V_{A A 2}^{2}}{\left[(l+p)^{2}-m_{A}^{2}\right]\left(l^{2}-m_{2}^{2}\right)}\right] \\
& +\left[\frac{2 V_{A A 11}}{l^{2}-m_{1}^{2}}+\frac{2 V_{A A 22}}{l^{2}-m_{2}^{2}}+\frac{12 V_{A A A A}}{l^{2}-m_{A}^{2}}\right],
\end{aligned}
$$

- The 1PI vertex corrections:

$$
\begin{aligned}
& -i \Delta V_{A A 1}=\int \frac{d^{4} l}{(2 \pi)^{4}}\left[\frac{6 V_{111} V_{A A 11}}{\left(l^{2}-m_{1}^{2}\right)^{2}}+\frac{2 V_{112} V_{A A 12}}{\left(l^{2}-m_{1}^{2}\right)\left(l^{2}-m_{2}^{2}\right)}\right. \\
& \left.+\frac{2 V_{122} V_{A A 22}}{\left(l^{2}-m_{2}^{2}\right)^{2}}+\frac{12 V_{A A 1} V_{A A A A}}{\left(l^{2}-m_{A}^{2}\right)^{2}}\right] \\
& +2 \times\left[\frac{4 V_{A A 1} V_{A A 11}}{\left[(l+p)^{2}-m_{A}^{2}\right]\left(l^{2}-m_{1}^{2}\right)}+\frac{2 V_{A A 2} V_{A A 12}}{\left[(l+p)^{2}-m_{A}^{2}\right]\left(l^{2}-m_{2}^{2}\right)}\right] \\
& +\left[\frac{12 V_{111} V_{A A 1}^{2}}{\left[(l+p)^{2}-m_{A}^{2}\right]\left(l^{2}-m_{1}^{2}\right)^{2}}+\frac{2 \times 4 V_{112} V_{A A 1} V_{A A 2}}{\left[(l+p)^{2}-m_{A}^{2}\right]\left(l^{2}-m_{1}^{2}\right)\left(l^{2}-m_{2}^{2}\right)}\right. \\
& \left.+\frac{4 V_{122} V_{A A 2}^{2}}{\left[(l+p)^{2}-m_{A}^{2}\right]\left(l^{2}-m_{2}^{2}\right)^{2}}\right] \\
& +\left[\frac{4 V_{A A 1}^{3}}{\left[(l+p)^{2}-m_{A}^{2}\right]^{2}\left(l^{2}-m_{1}^{2}\right)}+\frac{4 V_{A A 1} V_{A A 2}^{2}}{\left[(l+p)^{2}-m_{A}^{2}\right]^{2}\left(l^{2}-m_{2}^{2}\right)}\right], \\
& -i \Delta V_{A A 2}=\int \frac{d^{4} l}{(2 \pi)^{4}}\left[\frac{2 V_{112} V_{A A 11}}{\left(l^{2}-m_{1}^{2}\right)^{2}}+\frac{2 V_{122} V_{A A 12}}{\left(l^{2}-m_{1}^{2}\right)\left(l^{2}-m_{2}^{2}\right)}\right. \\
& \left.+\frac{6 V_{222} V_{A A 22}}{\left(l^{2}-m_{2}^{2}\right)^{2}}+\frac{12 V_{A A 2} V_{A A A A}}{\left(l^{2}-m_{A}^{2}\right)^{2}}\right] \\
& +2 \times\left[\frac{2 V_{A A 1} V_{A A 12}}{\left[(l+p)^{2}-m_{A}^{2}\right]\left(l^{2}-m_{1}^{2}\right)}+\frac{4 V_{A A 2} V_{A A 22}}{\left[(l+p)^{2}-m_{A}^{2}\right]\left(l^{2}-m_{2}^{2}\right)}\right] \\
& +\left[\frac{4 V_{112} V_{A A 1}^{2}}{\left[(l+p)^{2}-m_{A}^{2}\right]\left(l^{2}-m_{1}^{2}\right)^{2}}+\frac{2 \times 4 V_{122} V_{A A 1} V_{A A 2}}{\left[(l+p)^{2}-m_{A}^{2}\right]\left(l^{2}-m_{1}^{2}\right)\left(l^{2}-m_{2}^{2}\right)}\right. \\
& \left.+\frac{12 V_{222} V_{A A 2}^{2}}{\left[(l+p)^{2}-m_{A}^{2}\right]\left(l^{2}-m_{2}^{2}\right)^{2}}\right] \\
& +\left[\frac{4 V_{A A 1}^{2} V_{A A 2}}{\left[(l+p)^{2}-m_{A}^{2}\right]^{2}\left(l^{2}-m_{1}^{2}\right)}+\frac{4 V_{A A 2}^{3}}{\left[(l+p)^{2}-m_{A}^{2}\right]^{2}\left(l^{2}-m_{2}^{2}\right)}\right],
\end{aligned}
$$

Note that we have kept the momentum $p$ for external DM states while defining $\Delta V_{A A 1}$ and $\Delta V_{A A 2}$. The above 1PI irreducible diagrams are the basic ingredients for constructing more elaborated one-loop Feynman diagrams.

First of all, it is easy to write down the contributions to $\mathcal{F}$ from the one-loop external $A$ corrections shown in figure 5

$$
\mathcal{F}_{e}=\frac{2 i}{p^{2}-m_{A}^{2}}\left[-i \Delta m_{A}^{2}+\frac{2 i V_{A A 1} \Delta t_{1}}{m_{1}^{2}}+\frac{2 i V_{A A 2} \Delta t_{2}}{m_{2}^{2}}\right] \mathcal{F}_{0}=0
$$

where we have kept the same external $A$ momentum, $p$, which implies that the limit of zero momentum transfer was assumed. 


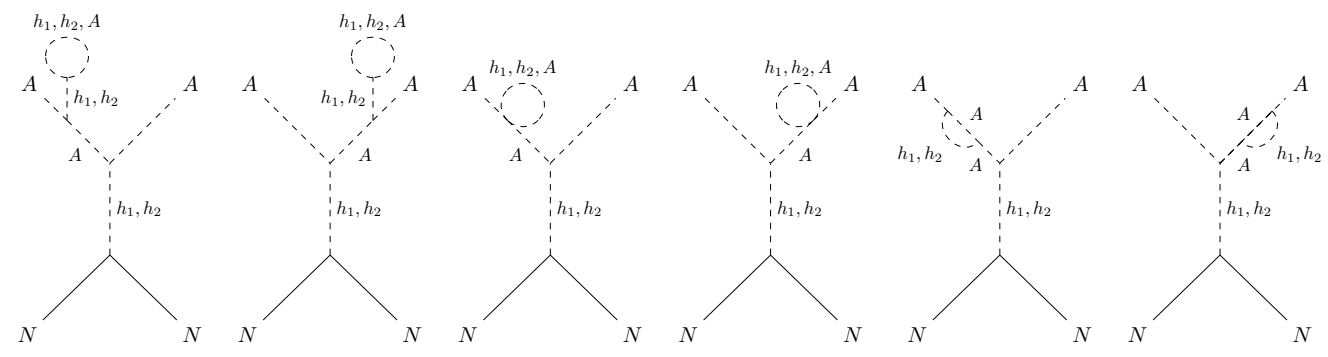

Figure 5. One-loop diagrams with external $A$-line corrections.

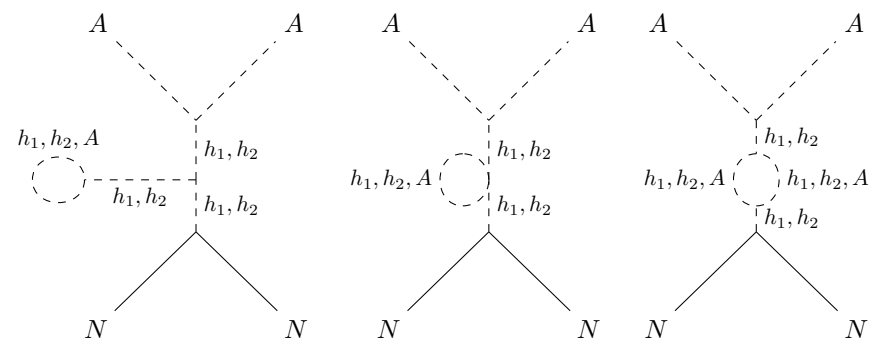

Figure 6. One-loop diagrams with internal $h_{1,2}$-propagator corrections.

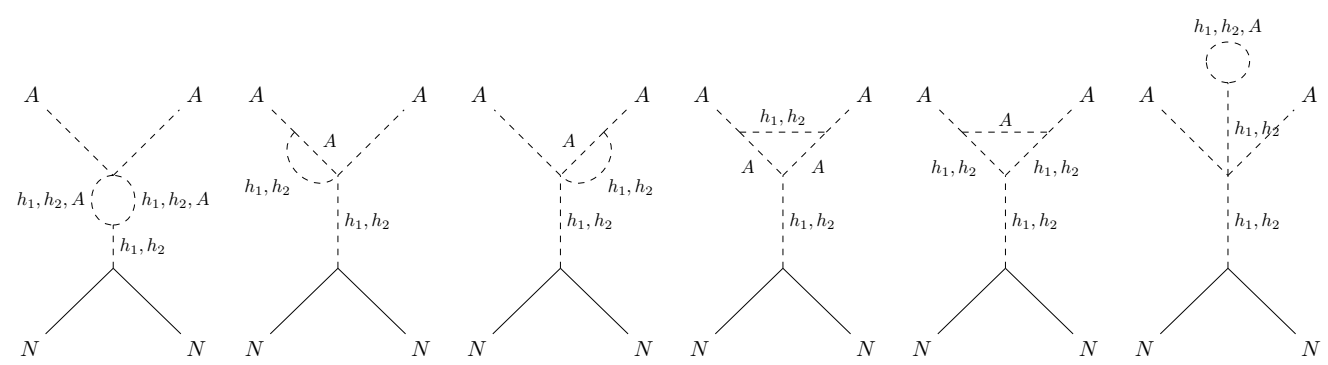

Figure 7. One-loop diagrams with vertices $A A h_{1}$ and $A A h_{2}$ corrections.

The remaining one-loop contributions are shown in figures 6 and 7 . The reducible contributions to the vertices $A A h_{1}$ and $A A h_{2}$ due to the internal $h_{1,2}$-propagator corrections are given by

$$
\begin{aligned}
-i V_{A A 1 i}^{(1)}= & i V_{A A 1} \frac{\Delta m_{1}^{2}}{m_{1}^{2}}+i V_{A A 2} \frac{\Delta m_{12}^{2}}{m_{2}^{2}} \\
& -\frac{6 i V_{A A 1} V_{111} \Delta t_{1}}{m_{1}^{4}}-\frac{2 i V_{A A 1} V_{112} \Delta t_{2}}{m_{1}^{2} m_{2}^{2}}-\frac{2 i V_{A A 2} V_{112} \Delta t_{1}}{m_{1}^{2} m_{2}^{2}}-\frac{2 i V_{A A 2} V_{122} \Delta t_{2}}{m_{2}^{4}}, \\
-i V_{A A 2 i}^{(1)}= & i V_{A A 1} \frac{\Delta m_{12}^{2}}{m_{1}^{2}}+i V_{A A 2} \frac{\Delta m_{2}^{2}}{m_{2}^{2}} \\
& -\frac{2 i V_{A A 1} V_{112} \Delta t_{1}}{m_{1}^{4}}-\frac{2 i V_{A A 1} V_{122} \Delta t_{2}}{m_{1}^{2} m_{2}^{2}}-\frac{2 i V_{A A 2} V_{122} \Delta t_{1}}{m_{1}^{2} m_{2}^{2}}-\frac{6 i V_{A A 2} V_{222} \Delta t_{2}}{m_{2}^{4}},
\end{aligned}
$$

while the ones from the vertex corrections are as follows:

$$
\begin{aligned}
& -i V_{A A 1 v}^{(1)}=-i \Delta V_{A A 1}+\frac{2 i V_{A A 11} \Delta t_{1}}{m_{1}^{2}}+\frac{i V_{A A 12} \Delta t_{2}}{m_{2}^{2}}, \\
& -i V_{A A 2 v}^{(1)}=-i \Delta V_{A A 2}+\frac{i V_{A A 12} \Delta t_{1}}{m_{1}^{2}}+\frac{2 i V_{A A 22} \Delta t_{2}}{m_{2}^{2}} .
\end{aligned}
$$


Thus, the total one-loop contribution to the factor $\mathcal{F}$ is given by

$$
\begin{aligned}
\mathcal{F}= & \frac{\left(V_{A A 1 i}^{(1)}+V_{A A 1 v}^{(1)}\right) c_{\alpha}}{m_{1}^{2}}-\frac{\left(V_{A A 2 i}^{(1)}+V_{A A 2 v}^{(1)}\right) s_{\alpha}}{m_{2}^{2}} \\
= & \frac{i s_{2 \alpha}\left(m_{1}^{2}-m_{2}^{2}\right)}{8 v_{H} v_{S}^{3} m_{1}^{2} m_{2}^{2}} \int \frac{d^{4} l}{(2 \pi)^{4}}\left[\frac{\mathcal{A}_{1}(l \cdot p)}{\left(l^{2}-m_{1}^{2}\right)\left(l^{2}-m_{2}^{2}\right)\left[(l+p)^{2}-m_{A}^{2}\right]}\right. \\
& \left.\quad+\frac{\mathcal{A}_{2}(l \cdot p)}{\left(l^{2}-m_{1}^{2}\right)^{2}\left(l^{2}-m_{2}^{2}\right)\left[(l+p)^{2}-m_{A}^{2}\right]}+\frac{\mathcal{A}_{3}(l \cdot p)}{\left(l^{2}-m_{1}^{2}\right)\left(l^{2}-m_{2}^{2}\right)^{2}\left[(l+p)^{2}-m_{A}^{2}\right]}\right]
\end{aligned}
$$

where the coefficients $\mathcal{A}_{i}$ are defined as follows

$$
\begin{aligned}
& \mathcal{A}_{1} \equiv 4\left(m_{1}^{2} s_{\alpha}^{2}+m_{2}^{2} c_{\alpha}^{2}\right)\left(2 m_{1}^{2} v_{H} s_{\alpha}^{2}+2 m_{2}^{2} v_{H} c_{\alpha}^{2}-m_{1}^{2} v_{S} s_{2 \alpha}+m_{2}^{2} v_{S} s_{2 \alpha}\right), \\
& \mathcal{A}_{2} \equiv-2 m_{1}^{4} s_{\alpha}\left[\left(m_{1}^{2}+5 m_{2}^{2}\right) v_{S} c_{\alpha}-\left(m_{1}^{2}-m_{2}^{2}\right)\left(v_{S} c_{3 \alpha}+4 v_{H} s_{\alpha}^{3}\right)\right], \\
& \mathcal{A}_{3} \equiv 2 m_{2}^{4} c_{\alpha}\left[\left(5 m_{1}^{2}+m_{2}^{2}\right) v_{S} s_{\alpha}-\left(m_{1}^{2}-m_{2}^{2}\right)\left(v_{S} s_{3 \alpha}+4 v_{H} c_{\alpha}^{3}\right)\right] .
\end{aligned}
$$

Note that in the derivation of eq. (4.21) we have used the tree-level relations from eq. (2.7) and the DM particle on-shell condition $p^{2}=m_{A}^{2}$.

We can utilize the Passarino-Veltman $C$ and $D$ functions as defined in refs. [24-26] to further reduce the expression of $\mathcal{F}$ to be

$$
\begin{aligned}
\mathcal{F}=-\frac{s_{2 \alpha}\left(m_{1}^{2}-m_{2}^{2}\right)}{128 \pi^{2} v_{H} v_{S}^{3} m_{1}^{2} m_{2}^{2}} p^{\mu}[ & \mathcal{A}_{1} C_{\mu}\left(0, p^{2}, p^{2}, m_{1}^{2}, m_{2}^{2}, m_{A}^{2}\right) \\
& +\mathcal{A}_{2} D_{\mu}\left(0,0, p^{2}, p^{2}, 0, m_{A}^{2}, m_{1}^{2}, m_{1}^{2}, m_{2}^{2}, m_{A}^{2}\right) \\
& \left.+\mathcal{A}_{3} D_{\mu}\left(0,0, p^{2}, p^{2}, 0, m_{A}^{2}, m_{1}^{2}, m_{2}^{2}, m_{2}^{2}, m_{A}^{2}\right)\right] \\
=-\frac{s_{2 \alpha}\left(m_{1}^{2}-m_{2}^{2}\right) m_{A}^{2}}{128 \pi^{2} v_{H} v_{S}^{3} m_{1}^{2} m_{2}^{2}}\left[\mathcal{A}_{1} C_{2}\left(0, m_{A}^{2}, m_{A}^{2}, m_{1}^{2}, m_{2}^{2}, m_{A}^{2}\right)\right. & \\
& +\mathcal{A}_{2} D_{3}\left(0,0, m_{A}^{2}, m_{A}^{2}, 0, m_{A}^{2}, m_{1}^{2}, m_{1}^{2}, m_{2}^{2}, m_{A}^{2}\right) \\
& \left.+\mathcal{A}_{3} D_{3}\left(0,0, m_{A}^{2}, m_{A}^{2}, 0, m_{A}^{2}, m_{1}^{2}, m_{2}^{2}, m_{2}^{2}, m_{A}^{2}\right)\right]
\end{aligned}
$$

where we have used $p^{2}=m_{A}^{2}$ and the following identity

$$
C_{\mu}\left(0, p^{2}, p^{2}, m_{1}^{2}, m_{2}^{2}, m_{A}^{2}\right)=p_{\mu} C_{2}\left(0, p^{2}, p^{2}, m_{1}^{2}, m_{2}^{2}, m_{A}^{2}\right),
$$

as well as the similar identities for $D$ functions. As anticipated earlier, this expression shows that the one-loop DM-nucleon scattering amplitude is finite in the zero-momentumtransfer limit. Moreover, since $\mathcal{F}$ is proportional to $m_{A}^{2}$ and the $C_{2}$ and $D_{3}$ functions behave as constants in the limit $m_{A} \rightarrow 0$ (see appendix B for details), the amplitude vanishes (as expected) in the limit $m_{A} \rightarrow 0$. It is highly non-trivial to satisfy both conditions at the same time, therefore this is an important test of our results.

\section{$5 \quad$ Numerical studies}

Having the explicit expression of the one-loop DM-nucleon recoiling cross section $\sigma_{\text {AN }}^{(1)}$ in eq. (4.1) with its loop function $\mathcal{F}$ in eq. (4.23), we can calculate the magnitude of the DMnucleon cross section with typical model parameters. In this section, we take $v_{S}=1 \mathrm{TeV}$, 


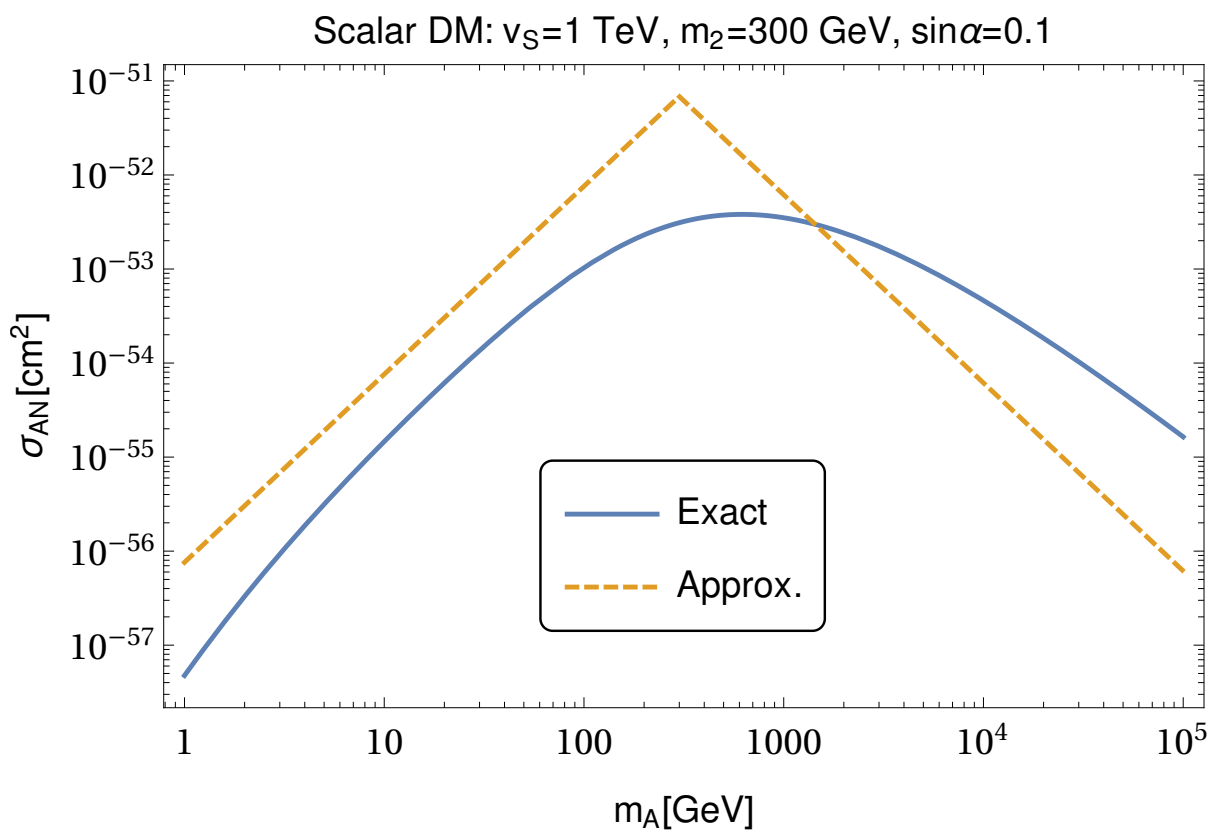

Figure 8. The DM-nucleon scattering cross section $\sigma_{\mathrm{AN}}$ as the function of the DM mass $m_{A}$. The blue solid curve represents the exact leading-order one-loop contribution in the limit of vanishing DM velocity, while the yellow dashed curve displays the approximate results proposed in ref. [10].

$m_{2}=300 \mathrm{GeV}, s_{\alpha}=0.1$, while leaving the DM mass varying freely. Note that we have reduced the final analytic expression for $\mathcal{F}$ in terms of the Passarino-Veltman functions, so that it is easy to calculate it numerically adopting the package LoopTools [26]. The final result is displayed in figure 8 as the smooth solid blue curve. We note that, for the given set of parameters, the DM-nucleon scattering cross section varies between $10^{-58} \mathrm{~cm}^{2}$ and $10^{-52} \mathrm{~cm}^{2}$ when the DM mass $m_{A}$ is in the range of $1-10^{5} \mathrm{GeV}$. For the same set of parameters the curve has a maximum value of $\sigma_{\mathrm{AN} \max }^{(1)} \sim 3 \times 10^{-53} \mathrm{~cm}^{2}$ for $m_{A} \sim 630 \mathrm{GeV}$. This should be compared with the tree-level contribution at the leading order of the DM velocity given in eq. (3.2), which predicts $\sigma_{\mathrm{AN}}^{\text {tree }} \sim 10^{-69}-10^{-65} \mathrm{~cm}^{2}$ with the same set of parameters. Thus, we can conclude that the leading-order DM-nucleon cross section is provided by the one-loop contributions at vanishing DM velocity, rather than the finite velocity corrections.

In contrast, we also show as the dashed yellow curve in figure 8 the following approximation proposed in ref. [10] as an estimate of the one-loop cross section

$$
\sigma_{\mathrm{AN}}^{(1)} \approx\left\{\begin{array}{l}
\frac{s_{\alpha}^{2}}{64 \pi^{5}} \frac{m_{N}^{4} f_{N}^{2}}{m_{1}^{4} v_{H}^{2}} \frac{m_{2}^{8}}{m_{A}^{2} v_{S}^{6}}, m_{A} \geq m_{2} \\
\frac{s_{\alpha}^{2}}{64 \pi^{5}} \frac{m_{N}^{4} f_{N}^{2}}{m_{1}^{4} v_{H}^{2}} \frac{m_{2}^{4} m_{A}^{2}}{v_{S}^{6}}, m_{A} \leq m_{2}
\end{array} .\right.
$$

It is clear that when $m_{A}$ lies below $1 \mathrm{TeV}$, the approximation is about one order larger than the exact result, while, if $m_{A} \gg 1 \mathrm{TeV}$, the exact $\sigma_{\mathrm{AN}}^{(1)}$ is almost one order higher. Nevertheless, these two curves share almost the same scaling behaviour in the limits of very small 


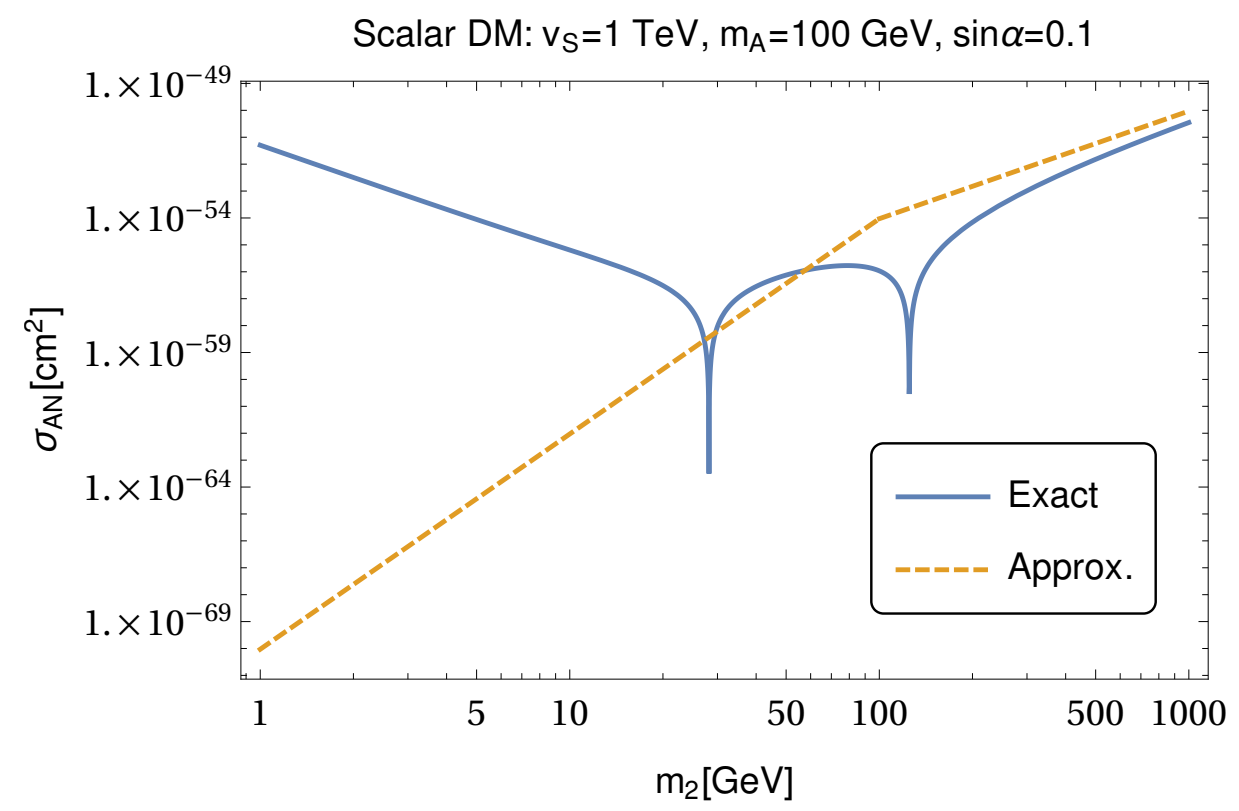

Figure 9. The DM-nucleon scattering cross section $\sigma_{\mathrm{AN}}$ as the function of $m_{2}$. The blue solid curve represents the exact leading-order one-loop contribution in the limit of vanishing DM velocity, while the yellow dashed curve displays the approximate results proposed in ref. [10].

and very large DM masses, which are reflected by the same slopes in the plot. Furthermore, both are well below the currently most stringent experimental limit of $\mathcal{O}\left(10^{-47}\right) \mathrm{cm}^{2}$ given by the XENON1T Collaboration. Therefore, the conclusion given in refs. $[10,12]$ that the DM direct detections does not impose any relevant constraints on the present model does not change. In particular, the available parameter space given in refs. $[10,12]$ is still the same, and would not change by using the exact formulae presented in eqs. (4.1) and (4.23) in the parameter scan.

In figure 9, we also show the DM-nucleon cross section as a function of the mass of non-SM-like Higgs boson $h_{2}$ with a fixed DM mass. We can see that the approximation, shown as the yellow dashed curve, substantially deviate from the exact formula in eqs. (4.1) and (4.23) drawn as the blue solid curve. The one-loop result shows much richer structure as the $h_{2}$ mass increases, rather than the simple scaling law predicted in eq. (5.1). In particular, two dips appear in the exact calculation. It is easy to see that one of them is located exactly at the point where $m_{2}=m_{1}$ corresponding to the vanishing of the factor $\left(m_{1}^{2}-m_{2}^{2}\right)$ in eq. (4.23). Another dip appearing at around $m_{2} \sim 30 \mathrm{GeV}$ is caused by accidental cancellation between loop integrals. The location of this dip varies with the set of parameters chosen and is a combination of all input parameters, the mass of the scalars, the angle $\alpha$ and $v_{S}$. Furthermore, note that when the $h_{2}$ mass is very small, the DM-nucleon cross section decreases as $m_{2}$ grows, in contrast with what is predicted by the approximate expression. On the other hand, when $m_{2}$ becomes much larger than the DM mass $m_{A}=100 \mathrm{GeV}$, the two curves approach each other, indicating that the approximation becomes valid only in this region. Finally we note that there is no difference between the approximate and the exact expression in the behaviour with the angle $\alpha$ and with the VEV $v_{S}$. 


\section{Conclusion}

In this work we have computed the one-loop electroweak contribution to DM-nucleon scattering, at zero momentum transfer, in a complex singlet extension of the SM with a softly broken $\mathrm{U}(1)$ symmetry. It has been shown in refs. $[10,12]$ that in such a simple extension of the SM with an extra complex scalar $S$ and with a softly broken U(1) symmetry, the pseudo-Goldstone component $A$ becomes the DM candidate and the tree-level contributions to the DM-nucleon recoiling cross section vanishes in the limit of zero momentum transfer. Therefore, the model has the attractive feature that the DM-nucleon cross section is naturally suppressed. Hence it is important to verify how large are one-loop contributions to the DM-nucleon scattering in this model. The calculation of these corrections, in the limit of zero momentum transfer, were the main goal of this work. We have shown that, for typical parameter choices, this one-loop contribution is 10 orders of magnitude larger than the finite-velocity or finite-momentum-transfer corrections at tree level. Therefore, we explicitly prove the expectation that the leading-order $\sigma_{\mathrm{AN}}$ indeed arises at the oneloop level. Furthermore, with the explicit analytic expression of $\sigma_{\text {AN }}$ given in eqs. (4.1) and (4.23), we show that the one-loop contribution is finite and approaches zero in the limit of vanishing DM mass. Finally, it has been shown that the DM-nucleon cross section is typically well below $\mathcal{O}\left(10^{-50} \mathrm{~cm}^{2}\right)$, which is much lower than the most stringent experimental upper bounds of $\mathcal{O}\left(10^{-47}\right)$ from XENON1T. This indicates that this model suppresses the DM direct detection signals so effectively that it is not constrained at all by this kind of experiments. Still, these radiative corrections will be important for the next generation of DM direct detection experiments, when the values of the cross sections that can be probed will reach the level of the one-loop result presented in this work. Finally we found accidental blind spots at the one-loop level, that is, points for which the DM-nucleon scattering cross section is still vanishingly small. These blind spots appear for given combination of parameters for which a next-order calculation would be needed.

\section{Acknowledgments}

This work is supported in part by the National Science Centre (Poland), research projects no 2014/15/B/ST2/00108, no 2017/25/B/ST2/00191, no 2017/25/N/ST2/01312 and a HARMONIA project under contract UMO-2015/18/M/ST2/00518 (2016-2019).

Note added: just after this paper had been submitted to the arXiv, another work [22] appeared with a calculation of the DM-nucleon cross section within the same model. In the high DM mass region our results qualitatively agree, with a deviation of at most a factor of two, but usually less, while the behaviour as a function of the DM mass shows a very similar trend. As we have checked, the discrepancy in the high DM mass region can not be attributed to a normalisation of the cross section. However it might be a consequence of different approaches adopted to calculate hadronic part of the cross section. On the other hand, in the low-DM-mass region, results from [22] predict growing DM-nucleon cross section with decreasing DM mass. This behaviour starts for DM masses of several tens of $\mathrm{GeV}$, as shown in their plot (left) in figure 2, and eventually tends to a constant 
when the DM mass becomes smaller than the nucleon mass. This is in clear contradiction with our result which shows the cross section approaching 0 as $m_{A} \rightarrow 0$. The authors of ref. [22] argue that this discrepancy is induced by the diagrams related to DM-gluon scattering shown as the second and fourth diagrams of the group (iii) in figure 1 in their paper. These diagrams are discarded in our calculation because they are subdominant as explained in section 4 . Therefore, the source of discrepancy should be located elsewhere. In particular, the low-DM-mass behaviour given in ref. [22] seems to be in conflict with the Goldstone nature of the DM particle in the zero-mass limit, which always predicts that the DM-nucleon scattering amplitude should be proportional to the momentum transfer squared and thus vanishes in the limit of zero momentum transfer. This conclusion should hold at any order of perturbation expansion. Our resulting cross section (4.1) and (4.23) indeed satisfies this requirement.

\section{A Tree-level interacting vertices}

By expanding the tree-level potential in eq. (2.1) in terms of the physical mass eigenstates $h_{1,2}$ and $A$, the tree-level triple- and quartic-scalar vertices can be written as follows,

$$
\begin{aligned}
\mathcal{V}_{\text {int }}= & V_{111} h_{1}^{3}+V_{112} h_{1}^{2} h_{2}+V_{122} h_{1} h_{2}^{2}+V_{222} h_{2}^{3}+V_{A A 1} h_{1} A^{2}+V_{A A 2} h_{2} A^{2} \\
& +V_{1111} h_{1}^{4}+V_{1112} h_{1}^{3} h_{2}+V_{1122} h_{1}^{2} h_{2}^{2}+V_{1222} h_{1} h_{2}^{3}+V_{2222} h_{2}^{4} \\
& +V_{A A 11} h_{1}^{2} A^{2}+V_{A A 12} h_{1} h_{2} A^{2}+V_{A A 22} h_{2}^{2} A^{2}+V_{A A A A} A^{4} .
\end{aligned}
$$

Note that only even powers of $A$ appear in the above interaction vertices which manifests the DM nature of $A$. The coefficients of the above vertices are listed below for reference,

$$
\begin{aligned}
V_{111} & =c_{\alpha}^{3} \lambda_{H} v_{H}+\frac{1}{2} s_{\alpha} c_{\alpha}^{2} \kappa v_{S}+\frac{1}{2} s_{\alpha}^{2} c_{\alpha} \kappa v_{H}+s_{\alpha}^{3} \lambda_{S} v_{S} \\
V_{112} & =\frac{1}{2} c_{\alpha}^{3} \kappa v_{S}+s_{\alpha} c_{\alpha}^{2} \kappa v_{H}-3 s_{\alpha} c_{\alpha}^{2} \lambda_{H} v_{H}-s_{\alpha}^{2} c_{\alpha} \kappa v_{S}+3 s_{\alpha}^{2} c_{\alpha} \lambda_{S} v_{S}-\frac{1}{2} s_{\alpha}^{3} \kappa v_{H} \\
V_{122} & =\frac{1}{2} c_{\alpha}^{3} \kappa v_{H}-s_{\alpha} c_{\alpha}^{2} \kappa v_{S}+3 s_{\alpha} c_{\alpha}^{2} \lambda_{S} v_{S}-s_{\alpha}^{2} c_{\alpha} \kappa v_{H}+3 s_{\alpha}^{2} c_{\alpha} \lambda_{H} v_{H}+\frac{1}{2} s_{\alpha}^{3} \kappa v_{S} \\
V_{222} & =c_{\alpha}^{3} \lambda_{S} v_{S}-\frac{1}{2} s_{\alpha} c_{\alpha}^{2} \kappa v_{H}+\frac{1}{2} s_{\alpha}^{2} c_{\alpha} \kappa v_{S}-s_{\alpha}^{3} \lambda_{H} v_{H} \\
V_{A A 1} & =s_{\alpha} \lambda_{S} v_{S}+\frac{1}{2} c_{\alpha} \kappa v_{H} \\
V_{A A 2}= & c_{\alpha} \lambda_{S} v_{S}-\frac{1}{2} s_{\alpha} \kappa v_{H} \\
V_{1111}= & \frac{1}{4}\left(c_{\alpha}^{4} \lambda_{H}+s_{\alpha}^{2} c_{\alpha}^{2} \kappa+s_{\alpha}^{4} \lambda_{S}\right) \\
V_{1112}= & \frac{1}{2} s_{\alpha} c_{\alpha}^{3} \kappa-s_{\alpha} c_{\alpha}^{3} \lambda_{H}-\frac{1}{2} s_{\alpha}^{3} c_{\alpha} \kappa+s_{\alpha}^{3} c_{\alpha} \lambda_{S} \\
V_{1122}= & \frac{1}{4}\left(c_{\alpha}^{4} \kappa+s_{\alpha}^{4} \kappa-4 \kappa s_{\alpha}^{2} c_{\alpha}^{2} \kappa+6 s_{\alpha}^{2} c_{\alpha}^{2} \lambda_{H}+6 s_{\alpha}^{2} c_{\alpha}^{2} \lambda_{S}\right) \\
V_{1222}= & -\frac{1}{2} s_{\alpha} c_{\alpha}^{3} \kappa+s_{\alpha} c_{\alpha}^{3} \lambda_{S}+\frac{1}{2} s_{\alpha}^{3} c_{\alpha} \kappa-s_{\alpha}^{3} c_{\alpha} \lambda_{H} \\
V_{2222}= & \frac{1}{4}\left(c_{\alpha}^{4} \lambda_{S}+s_{\alpha}^{2} c_{\alpha}^{2} \kappa+s_{\alpha}^{4} \lambda_{H}\right)
\end{aligned}
$$




$$
\begin{aligned}
V_{A A 11} & =\frac{1}{4}\left(2 s_{\alpha}^{2} \lambda_{S}+c_{\alpha}^{2} \kappa\right), \\
V_{A A 12} & =-\frac{1}{2} s_{\alpha} c_{\alpha} \kappa+s_{\alpha} c_{\alpha} \lambda_{S}, \\
V_{A A 22} & =\frac{1}{4}\left(2 c_{\alpha}^{2} \lambda_{S}+s_{\alpha}^{2} \kappa\right), \\
V_{A A A A} & =\frac{\lambda_{S}}{4}
\end{aligned}
$$

\section{B Analytic expressions of $C$ and $D$ functions}

In this appendix, we investigate behavior of the functions $C_{\mu}$ and $D_{\mu}$ utilized in (4.23):

$$
\begin{aligned}
C_{\mu} & =\int \frac{d^{4} l}{(2 \pi)^{4}} \frac{l_{\mu}}{\left(l^{2}-m_{1}^{2}\right)\left(l^{2}-m_{2}^{2}\right)\left[(l+p)^{2}-m_{A}^{2}\right]} \\
& =\frac{1}{m_{1}^{2}-m_{2}^{2}}\left(\mathcal{I}_{\mu}^{1}\left(m_{1}^{2}, m_{A}^{2}\right)-\mathcal{I}_{\mu}^{1}\left(m_{2}^{2}, m_{A}^{2}\right)\right),
\end{aligned}
$$

where the function $\mathcal{I}_{\mu}^{1}$ is defined as follows

$$
\begin{aligned}
\mathcal{I}_{\mu}^{1}\left(m_{1}^{2}, m_{A}^{2}\right) \equiv \int \frac{d^{4} l}{(2 \pi)^{4}} & \frac{l_{\mu}}{\left(l^{2}-m_{1}^{2}\right)\left[(l+p)^{2}-m_{A}^{2}\right]} \\
=-\frac{i p_{\mu}}{16 \pi^{2}} \frac{1}{2} & \left\{\left(\frac{2}{\epsilon}-\gamma+\ln \frac{\mu^{2}}{m_{A}^{2}}\right)\right. \\
& \left.\quad+\left[1+\left(x_{1}+x_{2}\right)+x_{1}^{2} \ln \frac{x_{1}-1}{x_{1}}+x_{2}^{2} \ln \frac{x_{2}-1}{x_{2}}\right]\right\},
\end{aligned}
$$

where we have defined the symbols $x_{1,2} \equiv\left(m_{1}^{2} \pm \sqrt{m_{1}^{4}-4 m_{1}^{2} m_{A}^{2}}\right) /\left(2 m_{A}^{2}\right)$ to denote the two roots of the equation $x^{2}-m_{1}^{2} x / m_{A}^{2}+m_{1}^{2} / m_{A}^{2}=0$. We also have used the dimensional regularisation to regularize the UV divergence in $\mathcal{I}_{\mu}^{1}$. Note that eq. (B.2) is only valid when $m_{1}^{2}>4 m_{A}^{2}$. It turns out that in the limit of $m_{A}^{2} / m_{1}^{2} \rightarrow 0, I_{\mu}^{1}\left(m_{1}^{2}, m_{A}^{2}\right)$ approaches

$$
I_{\mu}^{1}\left(m_{1}^{2}, m_{A}^{2}\right) \rightarrow-\frac{i p_{\mu}}{32 \pi^{2}}\left(\frac{2}{\epsilon}-\gamma+\ln \frac{\mu^{2}}{m_{1}^{2}}+\frac{3}{2}\right) .
$$

Therefore, the function $C_{\mu}$ (B.1) in the $m_{A}^{2} \rightarrow 0$ behaves as

$$
C_{\mu} \rightarrow \frac{i p_{\mu}}{32 \pi^{2}} \frac{\ln \left(m_{2}^{2} / m_{1}^{2}\right)}{m_{2}^{2}-m_{1}^{2}}
$$

which is obviously finite.

The $D_{\mu}$ functions in (4.23) could be investigated in a similar way. Let's start with

$$
\begin{aligned}
D_{\mu}\left(0,0, p^{2}, p^{2},\right. & \left.0, m_{A}^{2}, m_{1}^{2}, m_{1}^{2}, m_{2}^{2}, m_{A}^{2}\right) \\
& \equiv \int \frac{d^{4} l}{(2 \pi)^{4}} \overline{\left(l^{2}-m_{1}^{2}\right)^{2}\left(l^{2}-m_{2}^{2}\right)\left[(l+p)^{2}-m_{A}^{2}\right]} \\
& =\frac{1}{m_{1}^{2}-m_{2}^{2}}\left[\mathcal{I}_{\mu}^{2}\left(m_{1}^{2}, m_{A}^{2}\right)-C_{\mu}\left(0, p^{2}, p^{2}, m_{1}^{2}, m_{2}^{2}, m_{A}^{2}\right)\right],
\end{aligned}
$$


where

$$
\begin{aligned}
\mathcal{I}_{\mu}^{2}\left(m_{1}^{2}, m_{A}^{2}\right) & \equiv \int \frac{d^{4} l}{(2 \pi)^{4}} \frac{l_{\mu}}{\left(l^{2}-m_{1}^{2}\right)^{2}\left[(l+p)^{2}-m_{A}^{2}\right]} \\
& =-\frac{i p_{\mu}}{16 \pi^{2} m_{A}^{2}}\left[1+\frac{x_{1}\left(x_{1}-1\right)}{x_{1}-x_{2}} \ln \frac{x_{1}-1}{x_{1}}-\frac{x_{2}\left(x_{2}-1\right)}{x_{1}-x_{2}} \ln \frac{x_{2}-1}{x_{2}}\right],
\end{aligned}
$$

with $x_{1,2}$ defined above. By taking the zero-DM-mass limit, $\mathcal{I}_{\mu}^{2}\left(m_{1}^{2}, m_{A}^{2}\right)$ reduces to

$$
\mathcal{I}_{\mu}^{2}\left(m_{1}^{2}, m_{A}^{2}\right) \rightarrow \frac{i p_{\mu}}{32 \pi^{2} m_{1}^{2}}
$$

which could be also obtained by taking the limit of $m_{2}^{2} \rightarrow m_{1}^{2}$ in (B.4). Since the $C_{\mu}$ function is finite in the same limit (as it has been shown earlier), the $D_{\mu}$ function is also finite in this limit. Same arguments apply to the second $D_{\mu}$ function in (4.23).

Open Access. This article is distributed under the terms of the Creative Commons Attribution License (CC-BY 4.0), which permits any use, distribution and reproduction in any medium, provided the original author(s) and source are credited.

\section{References}

[1] Particle Data Group, Review of particle physics, Phys. Rev. D 98 (2018) 030001.

[2] L. Bergstrom, Dark matter evidence, particle physics candidates and detection methods, Annalen Phys. 524 (2012) 479 [arXiv: 1205.4882] [INSPIRE].

[3] G. Bertone, D. Hooper and J. Silk, Particle dark matter: evidence, candidates and constraints, Phys. Rept. 405 (2005) 279 [hep-ph/0404175] [INSPIRE].

[4] J.L. Feng, Dark matter candidates from particle physics and methods of detection, Ann. Rev. Astron. Astrophys. 48 (2010) 495 [arXiv: 1003.0904] [INSPIRE].

[5] XENON collaboration, Dark matter search results from a one ton-year exposure of XENON1T, Phys. Rev. Lett. 121 (2018) 111302 [arXiv:1805.12562] [INSPIRE].

[6] J. McDonald, Gauge singlet scalars as cold dark matter, Phys. Rev. D 50 (1994) 3637 [hep-ph/0702143] [INSPIRE].

[7] V. Barger et al., Complex singlet extension of the standard model, Phys. Rev. D 79 (2009) 015018 [arXiv: 0811.0393] [INSPIRE].

[8] V. Barger, M. McCaskey and G. Shaughnessy, Complex scalar dark matter vis-à-vis CoGeNT, DAMA/LIBRA and XENON100, Phys. Rev. D 82 (2010) 035019 [arXiv: 1005.3328] [INSPIRE].

[9] M. Gonderinger, H. Lim and M.J. Ramsey-Musolf, Complex scalar singlet dark matter: vacuum stability and phenomenology, Phys. Rev. D 86 (2012) 043511 [arXiv:1202.1316] [INSPIRE].

[10] C. Gross, O. Lebedev and T. Toma, Cancellation mechanism for dark-matter-nucleon interaction, Phys. Rev. Lett. 119 (2017) 191801 [arXiv:1708.02253] [INSPIRE].

[11] W. Cheng and L. Bian, From inflation to cosmological electroweak phase transition with a complex scalar singlet, Phys. Rev. D 98 (2018) 023524 [arXiv:1801.00662] [INSPIRE]. 
[12] D. Azevedo et al., Testing scalar versus vector dark matter, arXiv:1808.01598 [INSPIRE].

[13] XENON collaboration, Physics reach of the XENON1T dark matter experiment, JCAP 04 (2016) 027 [arXiv : 1512.07501] [INSPIRE].

[14] B.J. Mount et al., LUX-ZEPLIN (LZ) technical design report, arXiv:1703.09144 [INSPIRE].

[15] DARWIN collaboration, DARWIN: towards the ultimate dark matter detector, JCAP 11 (2016) 017 [arXiv : 1606. 07001] [INSPIRE].

[16] L. Baudis, Direct dark matter detection: the next decade, Phys. Dark Univ. 1 (2012) 94 [arXiv: 1211.7222] [INSPIRE].

[17] T. Han, H. Liu, S. Mukhopadhyay and X. Wang, Dark matter blind spots at one-loop, arXiv: 1810.04679 [INSPIRE].

[18] ATLAS, CMS collaboration, Measurements of the Higgs boson production and decay rates and constraints on its couplings from a combined ATLAS and CMS analysis of the LHC pp collision data at $\sqrt{s}=7$ and $8 \mathrm{TeV}$, JHEP 08 (2016) 045 [arXiv: 1606. 02266] [INSPIRE].

[19] J.M. Cline, K. Kainulainen, P. Scott and C. Weniger, Update on scalar singlet dark matter, Phys. Rev. D 88 (2013) 055025 [Erratum ibid. D 92 (2015) 039906] [arXiv:1306.4710] [INSPIRE].

[20] J.M. Alarcon, J. Martin Camalich and J.A. Oller, The chiral representation of the $\pi N$ scattering amplitude and the pion-nucleon sigma term, Phys. Rev. D 85 (2012) 051503 [arXiv:1110.3797] [INSPIRE].

[21] X.-L. Ren, X.-Z. Ling and L.-S. Geng, Pion-nucleon sigma term revisited in covariant baryon chiral perturbation theory, Phys. Lett. B 783 (2018) 7 [arXiv:1710.07164] [INSPIRE].

[22] K. Ishiwata and T. Toma, Probing pseudo Nambu-Goldstone boson dark matter at loop level, JHEP 12 (2018) 089 [arXiv: 1810.08139] [INSPIRE].

[23] A. Sirlin, Radiative corrections in the $\mathrm{SU}(2)-L \times \mathrm{U}(1)$ theory: a simple renormalization framework, Phys. Rev. D 22 (1980) 971 [INSPIRE].

[24] G. Passarino and M.J.G. Veltman, One loop corrections for $e^{+} e^{-}$annihilation into $\mu^{+} \mu^{-}$in the Weinberg model, Nucl. Phys. B 160 (1979) 151 [INSPIRE].

[25] A. Denner, Techniques for calculation of electroweak radiative corrections at the one loop level and results for W physics at LEP-200, Fortsch. Phys. 41 (1993) 307 [arXiv:0709.1075] [INSPIRE].

[26] T. Hahn and M. Pérez-Victoria, Automatized one loop calculations in four-dimensions and D-dimensions, Comput. Phys. Commun. 118 (1999) 153 [hep-ph/9807565] [INSPIRE]. 